\author{
Agnieszka Fus \\ ORCID: 0000-0003-2789-7500 \\ Universität Rzeszów, Rzeszów
}

DOI: $10.19195 / 0435-5865.143 .12$

\title{
Vulgarismen aus der Perspektive der pragmatischen Kontextabhängigkeit
}

\begin{abstract}
s
Im vorliegenden Beitrag wird der Versuch unternommen, anhand von zugänglichen Definitionen von Vulgarismen herauszufinden, warum manche Wörter als vulgär angesehen werden und aus welchen Themenbereichen sie stammen. Es wird eine eigene Terminologie vorgeschlagen, laut derer der Terminus Vulgarismus als Oberbegriff für die Begriffe Schimpfwort und Fluch fungiert. Näher betrachtet wird die Rolle, die der Kontext beim Auffassen von Vulgarismen spielt, die selten in ihrem literarischen oder wörtlichen Sinne benutzt werden. Darüber hinaus wird auf die Erscheinung des Bedeutungs- und Registerwandels aufmerksam gemacht, die im Fall von Vulgarismen besonders deutlich ist und in zwei Richtungen verläuft - entweder werden Wörter, die früher als vulgär galten, aufgewertet und nehmen eine neutrale oder sogar positive Bedeutung an, oder, ganz im Gegenteil, unterliegen Wörter, die früher neutral konnotiert waren, einer allmählichen Pejoration.
\end{abstract}

Schlüsselwörter: Vulgarismen, Kontext, interkulturelle Kommunikation

\section{The pragmatics of vulgarisms and swearing}

Swear words are well-suited to express emotion as their primary meanings are connotative. The emotional impact of swearing depends on one's experience with a culture and its language conventions. The present paper aims to compare the definitions of vulgarism in order to determine why some words are considered vulgar and from which their thematic areas originate. What is more, attention should be drawn to the phenomenon of change in meaning and register, which is particularly evident in the case of vulgarisms.

Keywords: vulgarisms, context, intercultural communication

Agnieszka Fus, Uniwersytet Rzeszowski, Instytut Filologii Germańskiej, Al. mjr. W. Kopisto 2b, 35-315 Rzeszów, Polen, E-Mail: agnieszka_fus@interia.pl

Received: 30.09.2017, accepted: 18.05.2018

Germanica Wratislaviensia 143, 2018

(C) for this edition by CNS 
Ich verstehe den Grundgedanken unanständiger Wörter nicht. Warum sind die Ausdrücke miteinander schlafen oder Geschlechtsverkehr haben annehmbar, während das Wort ficken, was das Gleiche bedeutet und ökonomischer ist, als unanständiges Schimpfwort gilt? Warum soll ein Wort, das Lust geben oder empfangen bedeutet, auch benutzt werden, um jemand zu beleidigen?

(Piercy, 2002: 128)

\section{Einleitendes}

Ihre Anwesenheit wird nicht selten von der Wissenschaft verschwiegen, sie stellen die sprachliche Unterwelt dar. Man beschuldigt sie der Verunstaltung einer Sprache. Manche sehen aber in ihnen ein einzigartiges Gewürz, das in kleinen Mengen einer Aussage einen besonderen Geschmack verleiht. Die meisten, die sie nicht verwenden, sind der Meinung, sie sollten aus allen Sprachsystemen verschwinden, vernichtet, ausgerottet werden. Die meisten, die sie verwenden, machen sich darüber überhaupt keine Gedanken. Vulgarismen und Kraftausdrücke, die den Gegenstand des vorliegenden Beitrags darstellen, ${ }^{1}$ existieren in allen Sprachen, ohne jegliche Ausnahme. Sie waren auch in Sprachen präsent, die es nicht mehr gibt. Dabei lässt sich nicht leugnen, dass sie, semantisch gesehen, völlig überflüssig und ersetzbar sind. Es muss ihnen irgendeine Rolle zustehen - davon könnten Menschen zeugen, in deren Aussagen sich immer Platz für Kraftausdrücke findet. ${ }^{2}$

\section{Zum Terminus Vulgarismus}

Jede Sprache enthält eine Sammlung von bestimmten Wörtern und Wendungen, deren Hauptfunktion das Ausdrücken von Emotionen ist, in Interaktion mit anderen häufig mit beleidigender, aggressiver Absicht. ${ }^{3}$ Welche Wörter es sind, hängt von der jeweiligen Kultur ab. Bei den einen großen Wert auf die Ästhetik legenden Japanern lautet die schlimmste Beleidigung ,Ich möchte den Bauchnabel deiner Mutter nicht sehen". Thailänder bezeichnen einen Menschen, den sie für dumm halten, als jemanden, mit dem ein Gespräch so viel Sinn hat wie Geige spielen für ein Nilpferd. In Westafrika drückt man sein Missfallen aus, indem man sagt: ,„Du stinkst wie ein Weißer unter der Achsel”. In Europa und Amerika dagegen greift

${ }^{1}$ Der vorliegende Text enthält einige Vulgarismen, die hier aber ausschließlich zu wissenschaftlichen Zwecken benutzt wurden.

${ }^{2}$ Nach einem Kinobesuch kann man doch sagen: "Der Film war miserabel" oder "Der Film war scheiße", ein unbeliebter Nachbar kann eine gemeine Person oder ein Ar...loch genannt werden.

${ }^{3}$ Ausführlicher dazu vgl. Bonacchi (2012 und 2014). 
man zu Vulgarismen (Hartwig 2003: 55). Crystal bezeichnet Vulgarismen als Tabuwörter, die man in der Alltagssprache im westlichen Kulturraum trifft. Sie gelten als obszön und drücken Emotionen wie Hass, Frustration, Feindseligkeit oder Überraschung aus. Die populärsten Ausdrücke bestehen aus einzelnen Wörtern oder kurzen Phrasen, die sich durch einen unterschiedlichen Grad von Intensität auszeichnen und deren Gebrauch negative Sanktionen nach sich ziehen kann (vgl. Crystal 1987: 61). Begriffe, die unterhalb der „Normalsprache” liegen, werden als salopp, derb, vulgär oder obszön beschrieben. Vulgärsprachliche Ausdrücke sind meist sehr anschaulich, spontan und konkret, aber auch brutal geprägt (Müller 2001: 13). In Polen stoßen die vulgären Ausdrücke auf Ablehnung und ihre Benutzer stellen sich durch deren Gebrauch häufig selbst ins Abseits. Puzynina (1994: 385) bezeichnet Wörter, die im Wörterbuch als vulgär markiert werden, als Wörter, die bei einem durchschnittlichen Zuhörer einen negativen Eindruck vom Sprecher hervorrufen. ,Trotz der öffentlich-gesellschaftlichen Nichtakzeptanz besitzen die Begriffe jedoch einen hohen Bekanntheitsgrad" (Kluge 1994: 159 f.).

Duden. Das große Wörterbuch der deutschen Sprache (1993-1995) definiert das Wort vulgär als „auf abstoßende Weise derb u. gewöhnlich, ordinär”. Demensprechend wird der Begriff Vulgarismus als „vulgäres Wort, vulgäre Wendung" definiert. Der Terminus Vulgarismus ist vielen deutschen Muttersprachlern nicht bekannt, als synonymer Begriff ist der Begriff Kraftausdruck bzw. Kraftwort geläufig, das Porzig (1971: 46) sehr zutreffend definiert als „einen derben, übertreibenden, bizarr-anschaulichen Ausdruck aus der niedrigen Umgangssprache, der eben dadurch imstande ist, ein Ding nicht nur zu benennen, sondern gleichzeitig die Gefühle des Sprechenden zu entladen".

Grochowski (2002: 18 ff.) betont in der Einleitung seines Buchs Stownik polskich przekleństw $i$ wulgaryzmów, dass sich Vulgarität nicht ausschließlich auf die Sprache bezieht, sie betrifft auch viele andere Gegenstände und Tätigkeiten, die zur außersprachlichen Realität gehören, wie z.B. sich anziehen oder tanzen, die aber nicht unabhängig von der Sprache erklärt werden können. Gemäß allgemein geltenden gesellschaftlichen Konventionen werden nicht alle Aspekte des menschlichen Lebens öffentlich kundgegeben, insbesondere diejenigen, die die Sphäre Sexualität betreffen. Die meisten Menschen sind sich dessen bewusst, dass der Gebrauch von manchen Wörtern und Wendungen, die negative Emotionen ausdrücken (es sind meistens Wörter, die Körperteile oder physiologische Aktivitäten wie Geschlechtsverkehr bezeichnen), verpönt ist. Man kann also vermuten, dass diejenigen, die sich der Sprache als Kommunikationsmittel bedienen, über eine gewisse „Autozensur" verfügen: sie wissen, dass sie mit Hilfe von bestimmten Wörtern in bestimmten Kontexten, die allgemein in der jeweiligen Gesellschaft akzeptierten Normen verletzen. Zu dem lexikalischen System jeder Sprache gehören sprachliche Einheiten, die mit einem Tabu belegt sind. Ein Vulgarismus ist somit, laut Grochowski (2002: 18), eine lexikalische Einheit, mit deren Hilfe der Sprechende seine Emotionen jemandem oder etwas gegenüber ausdrückt und da- 
bei ein sprachliches Tabu bricht. Grochowski (ebd. 20) teilt Vulgarismen in zwei Gruppen ein:

- Systemvulgarismen - alle Ausdrücke, die aufgrund von bestimmten Buchstabenfolgen, aus denen sie bestehen, als vulgär betrachtet werden, unabhängig von dem Kontext, in dem sie gebraucht werden und unabhängig davon, ob sie eine Bedeutung haben oder ob sie in bestimmten Kontexten als semantisch leere Einheiten gebraucht werden z.B. Scheiße oder kur...;

- Referenzvulgarismen - Ausdrücke, die wegen ihrer Semantik, aber nur bezüglich bestimmter Bedeutungsbereiche tabuisiert sind, z.B. walić oder Ritze.

Vulgarismen stellen lexikalische Mittel dar, die in der Sprachhandlung des Schimpfens und Fluchens Anwendung finden. Anderson und Trudgill (1990: 53) definieren Fluchen als ein Sprachverhalten mit expressiver Funktion, durch das man ein gesellschaftliches Tabu bricht. Das Fluchen könne nicht wörtlich interpretiert werden und diene zum Ausdruck von starken Emotionen und der persönlichen Meinung. Die beiden Forscher entwickeln im Rahmen ihrer Studie des Fluchens und Schimpfens eine pragmatisch orientierte Subdifferenzierung von Vulgarismen, die vier Kategorien umfasst:

- Vulgarismen, die zum Ausdruck von nicht personal adressierten Emotionen dienen (z.B. „Kur... mać, dlaczego to zepsułeś!?”; „Verdammte Scheiße, wir haben gewonnen!";

- Vulgarismen, die sich auf andere Personen beziehen: („I po coś tu przyszedł, chu... głupi!?”; „Du bist aber ein Ar...loch!”);

- Vulgarismen, die als Scherz gemeint sind, können auch andere Personen betreffen, werden aber nicht als beleidigend verstanden. Sie haben aber nur dann die Funktion einer witzigen Äußerung, wenn sich alle Gesprächsteilnehmer gut kennen und verstehen (z.B. „Rusz dupsko, bo nie mamy czasu.”; „Nächstes Mal kaufst du Bier, du Ar....");

- Vulgarismen als Füllwörter oder Ersatz für andere Wörter, sie beziehen sich weder auf Personen noch auf konkrete Situationen, für viele sind sie ein fester Bestandteil des Idiolekts (z.B. „Ten pier... autobus znów się spóźnia”; „Und wo sind die Scheißschuhe?".

Was die von Grochowski vorgeschlagene Subdifferenzierung von Vulgarismen betrifft, so lässt sich im Lichte der von Andersson und Trudgill (1990) entwickelten Kategorien feststellen, dass in jeder der vier Andersson-Trudgill-Kategorien sowohl System- als auch Referenzvulgarismen auftauchen können. Aus Gründen der analytischen Schärfe soll im folgenden Beitrag unter dem Oberbegriff Vulgarismus (bzw. synonym dazu Kraftausdruck) zwischen Schimpfen und Fluchen ${ }^{4}$ unterschieden werden. Orientiert an Andersson und Trudgill soll mit Fluch bzw. Fluchen der emotionale Kommentar einer Situation, mit Schimpfwort bzw.

${ }^{4}$ Dabei soll angemerkt werden, dass in der Umgangssprache Schimpfwort und Fluch synonym gebraucht werden. Grochowski (2002: 21) plädiert für die Unterscheidung zwischen den beiden. 
Schimpfen der emotionale (teilweise als Beleidigung intendierte) Kommentar zu einer an- oder abwesenden Person oder zu einem Objekt verstanden werden. Der gemeinsame Nenner beider Kategorien und damit das wesensbestimmende Merkmal von Vulgarismen liegt im obszön konnotierten Tabubruch. An dieser Stelle soll allerdings darauf hingewiesen werden, dass es unmöglich ist, generell zu behaupten, alle Schimpfwörter und Flüche seien Vulgarismen und umgekehrt, es gibt nämlich Flüche und Schimpfwörter, die nicht vulgär sind bzw. keinen obszön konnotierten Tabubruch darstellen (z.B. ,Kurka wodna, znowu uciekł nam tramwaj”; „No i z czego się śmiejesz, ty matpo głupia?”). Zugleich gibt es, besonders im Polnischen, auch Vulgarismen, die keine Flüche oder Schimpfe sind, insofern sie weder eine Situation noch eine Person auf die erläuterte Art und Weise kommentieren, z.B. „Ktoś zaje... jej portfel w autobusie”; „W tym hotelu kur ... są wyjątkowo drogie" (Grochowski 2002: 22).

Vulgarismen werden als Ergebnis soziokultureller Regeln und Überzeugungen betrachtet (vgl. Jay 1992: 1 ff.). In jeder Kultur gebe es sprachliche Einheiten, die als anstößig gelten. Es hänge von der jeweiligen Kultur ab, ob Obszönität, Blasphemie, Skatologie, sexuelle Witze, Tabuwörter oder Soziolekte akzeptiert oder abgelehnt werden. Die jeweilige Kultur determiniert, ob die Anwendung von Vulgarismen überhaupt (vgl. Beispiel Japan) und wenn ja, in welchen Kontexten annehmbar ist. Daher ist es klar, dass in westlichen Ländern das Fluchen in Anwesenheit höher gestellter Personen oder in der Kirche inakzeptabel ist. Im Sozialisationsprozess wird nämlich gelernt, welche Konsequenzen das Verwenden von Vulgarismen in bestimmten Situationen hat, was auch einen Einfluss auf die Wortwahl hat.

\title{
3. Kontextabhängigkeit
}

\author{
„Znaleziono psa, wiadomość w smażalni - \\ kontekst... ma znaczenie" \\ (Hund gefunden, Auskunft in der Grillstube - \\ der Kontext... hat Bedeutung)
}

(Demotywatory)

Vulgarismen zeichnen sich durch räumlich-zeitlich-kulturelle und soziale Kontextabhängigkeit aus, die einerseits bestimmt, ob ein Wort überhaupt als Vulgarismus gemeint bzw. verstanden wird (vgl. Grochowski, Referenzvulgarismen), andererseits, ob ein Vulgarismus zur Kommentierung einer Person als Beleidigung intendiert oder verstanden wird oder nicht (vgl. Andersson/Trudgill, Kategorien der Vulgarismen). Wie schon angedeutet, muss der Gebrauch eines Kraftausdruckes selbst nicht beleidigend sein. Und umgekehrt, kann man jemanden verletzen, ohne 
ein derbes Wort zu verwenden. ${ }^{5}$ Der Kontext, in dem ein vulgärer Ausdruck verwendet wird, hat Einfluss darauf, wie man die Intention des Sprechers versteht und ermöglicht entsprechende Reaktionen. Der Kontext ist von Belang, wenn man bestimmen muss, in welcher Absicht ein Kraftausdruck benutzt wird. Der Ort, die Umstände, die Beziehung und die Einstellung des Gesprächspartners entscheiden, wie die vulgäre Aussage interpretiert wird. Eine gewisse Rolle spielen dabei auch der Tonfall und die Intonation. In vielen Fällen ist der Kontext der einzige Faktor, der bestimmt, ob ein Wort als Beleidigung benutzt und verstanden wird oder nicht. Es ist ein großer Unterschied, ob man eine Hündin auf Polnisch als suka bezeichnet oder ob man so eine Arbeitskollegin nennt. Das Wort Schwein ist gar nicht beleidigend oder vulgär, wenn es ein Tier bezeichnet. Im Zusammenhang mit einer Person bedeutet das Benutzen dieses Wortes jedoch einen Verstoß gegen allgemein anerkannte gesellschaftliche Konventionen, hier wird es als beleidigend rezipiert. Ein ganz anderes Gewicht hat das Wort ficken, je nach dem, ob man es im lockeren Gespräch mit Freunden oder in der Anwesenheit der Großeltern verwendet. Im südländischen Kulturraum ist beispielsweise üblich und akzeptabel, sich unter Freunden vulgär zu beschimpfen, um die eigene Männlichkeit zu beweisen (vgl. ebd. 50). Der Eindruck, den man hinterlässt, indem man derben Wortschatz benutzt, wird zweifelsohne auch von dem Ort beeinflusst, an dem man flucht. Im westlichen Kulturraum gilt es als völlig unangebracht, deftige Ausdrücke in einem Amt, in der Kirche, Schule oder im Fernsehen zu verwenden. Zu Hause dagegen, im Freundeskreis oder in einer Bar hören sie sich nicht unbedingt fehl am Platz an. Die Rolle, die dem Kontext bei der Rezeption und Interpretation von Vulgarismen zukommt, wird zusätzlich durch die Tatsache verstärkt, dass sie meistens nicht wörtlich interpretiert werden können. Was genau darunter gemeint ist, kann am Beispiel des Wortes Scheiße erklärt werden. Es bezieht sich auf ein in unserer Kultur tabuisiertes Objekt, und zwar auf Exkremente, aber jemand, der fluchtend dieses Wort verwendet, denkt nicht an Ausscheidungsprodukte, er drückt einfach seinen Zorn, seine Verwunderung u.ä. aus (ebd. 53). Man könnte sogar die Behauptung wagen, dass Vulgarismen, ähnlich wie Phraseologismen, sich durch Idiomatizität auszeichnen. Die Bedeutung eines Phraseologismus hat mit Bedeutung seiner einzelnen Komponenten nichts zu tun. Ein ähnliches Phänomen können wir im Fall der Vulgarismen feststellen, bei deren Gebrauch selten die wörtliche Bedeutung gemeint ist - ein Schüler, der eine schlechte Note bekommt und deshalb seine Lehrerin $k u r . .$. nennt, findet diese Bezeichnung zwar durchaus negativ, behauptet aber nicht, dass er die beschimpfte Person für eine Prostituierte hält. Dieses Wort steht nämlich für negative Eigenschaften wie Boshaftigkeit, Gemeinheit u.ä. Es wird in dem bereits angeführten Beispiel auf diese Art und Weise verstanden. Andersson und Trudgill (1990: 59) unternehmen in ihrem Buch Bad language den Versuch, das Phänomen zu benennen, und einigen sich schließlich auf den

\footnotetext{
5 Vgl. auch: Bąk (2017).
} 
vielleicht nicht völlig angemessenen (wie sie selbst zugeben), aber die Sache auf den Punkt bringenden Terminus Metapher. Tatsächlich werden Vulgarismen in den meisten Fällen in übertragener Bedeutung gebraucht, wobei sich ihre eigentliche Bedeutung mit der Zeit modifizierte. Das Ganze kann man mit Hilfe eines einfachen Beispiels verdeutlichen, die drei folgenden Ausdrücke haben, wörtlich gesehen, ganz unterschiedliche Bedeutungen, in übertragenem Sinne aber, wenn sie im gleichen Kontext verwendet werden, bedeuten sie dasselbe: Spier ...! Zabieraj d...! Idź w pi...! (Grochowski 2002). Wie Grochowski (2002) behauptet, betrifft mindestens eine der Bedeutungen von jedem vulgären Hauptbegriff im Polnischen einen intimen Körperteil, Fäkalien oder sexuellen Akt. Sie beziehen sich also auf einen Lebensbereich, über den in der Öffentlichkeit nicht gesprochen wird. Aber auch in der deutschen Sprache handelt es sich bei den Vulgarismen hauptsächlich um Wörter, die den menschlichen Körper und seine Physiologie betreffen. Man kann aber nicht definitiv feststellen, dass diejenigen, die den sexuellen Wortschatz benutzen, zwangsläufig über Sex sprechen - jemand, der das Wort Schlappschwanz benutzt, macht in den meisten Fällen keine Anspielung auf die sexuelle Potenz eines Mannes, es wird damit eher eine Person mit bestimmten negativen Charakterzügen gemeint (vgl. Januschek 1986: 5).

\section{Bedeutungs- und/oder Registerwandel}

Man kann die Feststellung wagen, dass es keine sprachliche Norm gibt, die für immer gelten würde. Ein Beweis dafür sind Wörter, die früher eine völlig neutrale Bedeutung hatten, mittlerweile aber zum unanständigen Wortschatz gehören oder umgekehrt (vgl. Wiśniewska 1994: 82). Vulgärsprachliche Begriffe erfahren immer wieder Auf- bzw. Abwertungen (Frömel/Hopp 1996: 73). Ein Beispiel dafür liefert das polnische Wort kutas, das in früheren Zeiten eine Quaste bezeichnete und heutzutage ausschließlich als vulgäre Bezeichnung für Penis gebraucht wird. Hier liegt also sowohl ein Bedeutungs- als auch ein Registerwandel vor. Kutas ist ein Beispiel eines diachronen Referenzvulgarismus, der auf der Ebene der formalen Ähnlichkeit des Bezeichneten funktioniert. Ein deutsches Beispiel, das zu dieser Kategorie gehört, ist das Verb wichsen (früher: 'Fußboden scheuern', 'Schuhe polieren'; heute: 'masturbieren'). Auch das Verb ficken hatte noch im Jahre 1906 keine sexuelle Bedeutung (,,ein Kind ficken”) und diente als Synonym für schlagen. Heute würde man das Wort Kinderficker in Wörterbüchern vergebens suchen, obwohl es in der Sprache existiert und mehrmals nachgewiesen wurde (vgl. Müller 2001: 20).

Diachrone Referenzvulgarismen können auf der Ebene Homophonie funktionieren. Auch hier beinhalten sie sowohl einen Bedeutung- als auch Registerwandel. Der Wandel wird vollzogen, wenn ein Wort mit Vorstellungsinhal- 
ten verbunden wird, die den Sinngehalt des Schlechten aufnehmen. Auf diese Weise können ursprünglich seriöse Wörter zu Schimpfwörtern werden (Kiener 1983: 15). Einer solchen Pejoration unterlag auch das Wort pedat, das aufgrund seiner phonetischen Ähnlichkeit mit dem Wort pederasta zur vulgären Bezeichnung für Homosexuelle wurde. Im Stownik gwary studenckiej aus dem Jahr 1994 steht das Stichwort pedat nur als eine umgangssprachliche Bezeichnung für eine der Spielkarten (Grabias u.a. 1994). Der Ausdruck Klöten diente früher als normalsprachliche Umschreibung für Hoden. Heutzutage gehört es der vulgärsprachlichen Schicht an. Wahrscheinlich rief der Begriff aufgrund seiner häufigen Verwendung immer mehr gefühlsbedingte negative Assoziationen hervor, was letztendlich zu seiner Abwertung führte (Müller 1995: 160). Der Vorgang kann auch in die Gegenrichtung verlaufen, d.h. Wörter, die früher als unanständig galten, werden nicht mehr als solche betrachtet wie z.B. das deutsche Wort geil, dass jetzt nicht mehr in erster Linie mit sexueller Gier assoziiert wird, sondern als Bezeichnung einer sehr positiven Eigenschaft fungiert und nicht mit dem Etikett unanständig versehen wird: Der Film war richtig geil. Um es zu überprüfen reicht es aus, in den Wörterbüchern nachzuschlagen, die im Laufe der letzten 50 Jahre herausgegeben wurden, so Grochowski (zit. n. Sowa 2006). Den früheren Ausgaben kann man entnehmen, dass solche Wörter wie cholera völlig inakzeptabel waren, heutzutage jedoch eher niemand Anstoß daran nimmt. Es funktioniert sehr einfach - immer mehr Menschen wiederholen bestimmte Ausdrücke so oft, dass sich ihre ursprünglich negative Bedeutung verwischt. Es lässt sich immer häufiger beobachten, dass Wörter, die früher als offensichtlich obszön galten, heute an ihrer Ausdruckskraft verlieren, sie werden nicht mehr negativ konnotiert oder aber in Extremfällen sogar als zunehmend positiv empfunden, wie das polnische Adjektiv zajebisty, das die gleiche Bedeutung wie das deutsche Adjektiv geil hat, gleichzeitig aber ein Derivat des Verbs $j e . .$. , einer der zwei vulgärsten Bezeichnungen für Geschlechtsverkehr in der polnischen Sprache. Im 17 Jh. bedeutete das Wort kobieta ('Frau'), im entsprechenden Kontext benutzt, Nutte. Bielski schrieb in Sejm niewieści: „Męże Was, ku większemu zelżeniu kobietami zowią" (zit. n. Cieślik 2004). Auch das Wort $d u$... verliert immer mehr an seiner Vulgarität, man hört es oft in positiver Bedeutung als Bezeichnung für eine attraktive Person des anderen Geschlechts. Und wenn wir sagen, dass das Wetter kiepska ist, kommt uns nie das Wort kiepska in den Sinn, das früher eine sehr vulgäre Bezeichnung für weibliche Geschlechtsorgane war (Cieślik 2004). Von den bereits dargestellten Phänomenen des zwiefachen Wandelns (Bedeutungs- und Registerwandelns) ist das Phänomen des einfachen Wandelns, d.h. des bloßen Registerwandelns, zu unterscheiden. „Worttabus sind kreativ. [...] Die Vermeidung bestimmter Wörter führt zwangsläufig zur Schaffung von Synonymen und neuen Wörtern" (Balle 1990: 27). Viele Wörter, die früher ohne jegliche Konnotationen benutzt werden konnten, gelten heutzutage als unanständig, insofern sie anstößige, unangenehme oder gefährliche 
Gedanken hervorrufen. „Bekanntlich sind die Namen für gewisse Körperteile verpönt und müssen durch andere Bezeichnungen ersetzt werden. Dieser Vorgang wiederholt sich aber beständig, denn es dauert nicht lange, dann sind auch die neuen Namen in guter Gesellschaft unmöglich, weil die Anstößigkeit ja nicht am Namen, sondern an der Sache hängt" (Porzig 1971: 45). So verlieren Euphemismen mit der Zeit ihren „Ersatzcharakter” und werden selbst tabu, indem sie die volle Bedeutung des Tabuwortes annehmen (Pei 1949: 237). Jay (1977: 237) nennt es „Euphemismus-Tabu-Zyklus”. Als Beispiel dafür führt Porzig (ebd.) das Wort Arsch an, dessen älteste nachweisbare Form orsos selbst als eine verhüllende Bezeichnung diente und das später durch Hintern und schließlich durch Gesäß ersetzt wurde. Auch die deutsche Entsprechung des polnischen kutas, also Schwanz, spielte früher eine verhüllende Rolle - im Deutschen Wörterbuch der Brüder Grimm finden wir folgenden Eintrag für Schwanz (geschrieben von Heyne im Jahre 1898): „Früher auch in ernsthafter, züchtiger Rede, also als verhüllend empfunden, in neuerer Zeit nur als derb scherzhafter Ausdruck geltend." Der Registerwandel ist auch in der Gegenrichtung möglich. Das Wort schwul für Homosexuelle war noch in den 80er Jahren als extrem beleidigend empfunden, mittlerweile gehört es zur Standardsprache ohne jegliche abwertende Bedeutung - Homosexuelle bezeichnen sich selbst sehr oft als schwul. Einer ähnlichen Entwicklung unterliegt das Wort Hure - für die meisten fungiert es immer noch als Schimpfwort, wird aber von den das älteste Gewerbe der Welt ausübenden Frauen nicht als solches betrachtet, wenn es von ihnen als Selbstbezeichnung gebraucht wird, was eine gewisse Aufwertung des Begriffs mit sich trägt (Hoberg/ Fährmann 2001: 90). Vulgarismen sind eine feste Komponente der Alltagssprache und dienen meistens dazu, Gefühle zu entladen. Aber ihr emotionaler sowie teilweise ihr semantischer Gehalt stellt keine feste Größe dar (Kiener 1983: 15). Sie werden häufig gebraucht, was oft zur Folge hat, dass sie sich schnell abnutzen und durch neue Gebilde ersetzt werden müssen, um die erzielte Wirkung zu erreichen. „Selbst die kräftigsten und auffälligsten Ausdrücke werden durch Nachahmung sehr rasch Schablone" (Porzig 1971: 252). Wahrscheinlich ist es einer der Gründe, warum in der polnischen Sprache so viele Varianten von einzelnen Vulgarismen existieren - damit die Wirkung nicht verloren geht, muss sie stets verstärkt werden. ${ }^{6}$ Derselbe Prozess, nur mit umgekehrtem Vorzeichen, zeigt sich bei Euphemismen. Durch häufigen Gebrauch büßen sie mit der Zeit ihre verhüllende Wirkung ein. Hughes, G. (1991: 236) vergleicht die Gültigkeit von Kraftausdrücken mit Modetrends und vertritt den Standpunkt, dass die Veränderungen im Bereich des vulgären Wortschatzes, denen jede Sprache ständig unterliegt, am deutlichsten sind.

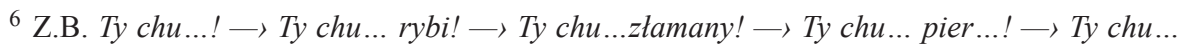
$w d u \ldots$ je...! usw. (Grochowski, 2002: 53 ff.) 


\section{Abschließendes und Ausblick}

Anhand der im Beitrag genannten Beispiele sollte verdeutlicht werden, dass die Bedeutung von Vulgarismen sehr stark von dem Anwendungskontext und pragmatischen Faktoren abhängt und nur im Zusammenhang mit diesen ergründet werden kann.

Darüber hinaus sollten wegen der starken Kontextgebundenheit von Vulgarismen bestimmten Kraftausdrücken auch keine Gemütszustände zugeschrieben werden, weil sie nicht dazu benutzt werden, um konkrete Informationen zu vermitteln. Das breite Spektrum an Emotionen, die sie ausdrücken, kann in weiteren wissenschaftlichen Überlegungen ergründet werden.

\section{Literatur}

Andersson, Lars-Gunnar / Trudgill, Peter (1990): Bad language. London.

Balle, Christel (1990): Tabus in der Sprache. Frankfurt am Main.

Bąk, Paweł (2017): Offene und versteckte Aggression im Gebrauch von Disphemismen und Euphemismen. In: Bonacchi, Silvid (Hrsg.): Verbale Aggression. Multidisziplinäre Zugänge zur verletzenden Macht der Sprache. Berlin. S. 127-143.

Bonacchi, Silvia (2012): Zu den idiokulturellen und polykulturellen Bedingungen von aggressiven Äußerungen im Vergleich Polnisch-Deutsch-Italienisch. In: Olpińska-Szkiełko, M. / Bartoszewicz M. / Grucza, E. (Hrsg.): Der Mensch und seine Sprache. Frankfurt am Main u.a. S. $130-148$.

Bonacchi, Silvia (2014): Scheinbeleidigungen und perfide Komplimente: kulturologische Bemerkungen zur obliquen Kommunikation in interkultureller Perspektive. In: Lukas, K. / Olszewska, I. (Hrsg.): Deutsch im Kontakt und im Kontrast. Festschrift für Andrzej Kątny zum 65. Geburtstag. Frankfurt am Main u.a. S. 341-356.

Crystal, David (1987): Child language, learning and linguistics: an overview for the teaching and therapeutic professions. London u.a.

Duden. Das große Wörterbuch der deutschen Sprache in acht Bänden. Mannheim 1993-1995.

Frömel, Kerstin / Hopp, Ursula (1996): Die Sexualsprache in Aufklärungsbüchern für Jugendliche (1960-1994). Textanalyse ausgewählter Sexualwörter. In: Kluge, N. (Hrsg.): Jugendliche Sexualsprache - eine gesellschaftliche Provokation. Landau. S. 69-136.

Grabias, Stanisław / Kaczmarek, Leon / Skubalanka, Teresa (1994): Stownik gwary studenckiej. Lublin.

Grimm Jacob und Willhelm (1854-1960): Deutsches Wörterbuch. 16 Bände. Leipzig.

Grochowski, Maciej (2002): Słownik polskich przekleństw i wulgaryzmów. Warszawa.

Hartwig, H. (2003): Rosót z kur wielu. Forum 27. S. 54-55.

Hoberg, Rudolf / Fährmann, Rosemarie (2001): Zur Sexualsprache der Studierenden. In: Hoberg, R. (Hrsg.): Sprache - Erotik - Sexualität. Berlin. S. 173-191.

Januschek, Franz (Hrsg.) (1986): Sprache und Sexualität. Bremen.

Hughes, Geoffrey (1991): Swearing. A Social History of Foul Language. Oaths and Profanity in English. Oxford/Cambridge.

Jay, Timothy (1997): Doing research with dirty words. In: Maledicta: The International Journal of Verba. Aggression 1. S. 234-256. 
Jay, Timothy (1992): Cursing in America. A psycholinguistic study of dirty language in the courts, in the movies, in the schoolyards and on the streets. Philadelphia/Amsterdam.

Kiener, Franz (1983): Das Wort als Waffe. Zur Psychologie der verbalen Aggression. Göttingen.

Kluge, Norbert (1994): Die Sexualsprache in Jugendbroschüren - Beobachtungen anhand zweier Neuerscheinungen. In: Sexuologie 3/1. Stuttgart. S. 159-166.

Müller, Wolfgang (2001): Seid reinlich bei Tage und säuisch bei Nacht (Goethe) oder: Betrachtungen über die schönste Sache der Welt im Spiegel der deutschen Sprache - einst und jetzt. In: Hoberg, R. (Hrsg.): Sprache - Erotik - Sexualität. Berlin. S. 11-61.

Pei, Mario (1949): The Story of Language. London.

Piercy, Marge (2002): Er, Sie und Es. Hamburg.

Porzig, Walter (1971): Das Wunder der Sprache. Probleme, Methoden und Ergebnisse der Sprachwissenschaft. Bern.

Puzynina, Jadwiga (1994): Refleksje dotyczace Suplementu do Stownika języka polskiego pod red. M. Szymczaka. In: Polszczyzna a/i Polacy u schyłku XX wieku. Warszawa.

Wiśniewska, Halina (1994): Co poczać z wulgaryzmami? In: Polonistyka 2. S. 3-9.

\section{Internetquellen}

Cieślik, Mariusz: Gięcie kobiet. In: Wprost 27, 2004. http://www.wprost.pl/ar/?O=62467\&C (29.08.2017).

Demotywatory: http://www.demotywatory.pl (28.08.2017).

Sowa, Sławomir: Rozmowa dnia. Prof. M. Grochowski: Dlaczego Polacy tak przeklinaja? http:// pomorskie.naszemiasto.pl/artykul/prof-maciej-grochowski-dlaczego-polacy-tak-przeklinaja-24,176951,art,t,id,tm.html (28.08.2017). 